\title{
Letter from the Editor: The IGS Special Issue
}

Jim Ray, Guest Editor

he International GPS

Service (IGS) began in 1992 as a collaboration among numerous national agencies and university groups to provide GPS data and orbits of the highest quality for very demanding geodetic applications. Originally, the IGS products were intended for geoscience users requiring differential positioning at the sub-cm level in a post-processing mode. With improvements in the global tracking network, data flow, and analysis, the IGS orbit error has dropped from $>20 \mathrm{~cm}$ in 1994 to $<5$ $\mathrm{cm}$ today. (Broadcast GPS orbits have an uncertainty of $>250 \mathrm{~cm}$.)

Past applications were satisfied with post-processing delays of days to weeks. However, the success of the IGS has sparked interest in a broader range of applications, especially near real-time uses. Examples include mapping variations in the Earth's ionosphere, tropospheric sensing for weather forecasting, global time and frequency transfer, and precisely navigating low Earth orbiting satellites. Increasingly, lower accuracy but more nearly real-time applications are becoming prominent. The most demanding requirements call for real-time (predicted) GPS orbits accurate at the decimeter level and satellite clocks at the few-nanosecond level. These are challenges that the IGS is currently striving to satisfy.

Against this backdrop, the IGS held its annual Analysis Center Workshop during 25-29 September 2000 at the U.S. Naval Observatory in Washington, DC. Some of the papers presented there are published in this special issue.

The IGS Ultra-rapid service, which began in November 2000 , aims to satisfy near real-time users, as described in the paper by Fang et al. These orbits are issued with only 3 hours' latency, including 24 hours of observed orbits together with 24 hours of predictions. They are updated twice daily so that the mean latency of the predictions is only 9 hours and the accuracy is about $20 \mathrm{~cm}$. The most demanding user communities for these products are meteorological forecasters of short-term weather. Gutman and Benjamin review the assimilation process for GPS-derived tropospheric soundings and the implications.

The potential interactions and opportunities between the IGS and various GNSS systems, particularly Europe's new Galileo constellation, are discussed by Willis and Dow. In response, a new IGS Working Group will be formed to provide a mechanism for ongoing information exchange. The considerations impacting the design of the Galileo constellation are reviewed by Mozo-Garcia et al. Significantly, the Galileo orbits will be higher than GPS to avoid 12-hour resonance and eliminate the need for station keeping maneuvers. The status of the existing IGS Pilot Project for GLONASS is given by Weber and Slater, who find that sufficient user interest exists to continue the project.

The progress of the IGS/BIPM Time Transfer Pilot Project is sur- veyed in the paper by Ray et al. The IGS has expanded its clock products to include receiver clocks as well satellites (see Kouba and Springer). Practical utilization will depend on the development of calibration methods that can be applied to geodetic receiver systems. Impressive achievements in this area are presented by Petit et al. Among the effects which must be considered and controlled in order to achieve the full potential of geodetic time transfer are observation biases. Jefferson et al. report their results in resolving satellite-based biases between the $\mathrm{C} / \mathrm{A}$ and codeless $\mathrm{Pl}$ pseudorange observables.

Among the other interesting work reported at the workshop are the papers by Mader and by Rothacher on new absolute antenna phase pattern results. While new measurements confirm the absolute phase patterns for most geodetic antennas, use of these patterns in GPS data analyses causes the terrestrial reference frame to shift by 15 ppb. This is implausible in view of the very accurate and consistent frames from independent techniques. The GPS scale can be reconciled if the properties of the satellite transmitter arrays are also considered, an effort currently under study.

Jim Ray, Ph.D., is the head of the Earth Orientation Department at the U.S. Naval Observatory. He also serves on the IGS Governing Board and acts as co-chair of the IGS/BIPM Time Transfer Pilot Project. 